Pacific Journal of Mathematics

GROUPS WHOSE IRREDUCIBLE REPRESENTATIONS HAVE Donald STEven TAsman 


\title{
GROUPS WHOSE IRREDUCIBLE REPRESENTATIONS HAVE DEGREES DIVIDING $p^{2}$
}

\author{
D. S. PASSMAN
}

In several previous papers I. M. Isaacs and this author studied properties of groups which are related to the degrees of their absolutely irreducible representations and in particular to the biggest such degree. The restilts were concerned mainly with the existence of "large" abelian subgroups in these groups. It was found that much more could be said in the $p$-group-like situation in which the degrees of the irreducible characters of group $G$ are all powers of a fixed prime $p$. We say group $G$ has r.x.e (representation exponent $e$ ) if the degrees of all the irreducible characters of $G$ divide $p^{e}$. In this paper we characterize groups with r.x.2. It is found that the prime $p=2$ plays a special role here. This supports the conjecture that additional and more complicated groups with r.x.e occur for $p \leqq e$. With a few exceptions for $p=2$, all groups $G$ with r.x.2 are shown to have either a normal subgroup of index $p$ with r.x.1 or a center of index dividing $p^{6}$.

1. Preliminary remarks. We will use here the notation and many of the results of the first four sections of [5]. For example, we need the characterization of groups with r.x.1 given there. From this we obtain the following.

(1.1) Lemma. (i) Let $N$ have r.x.1. Then either $N$ has a characteristic abelian subgroup of index $p$ or $[N: 3(N)]$ divides $p^{3}$ where $3(N)$ denotes the center of $N$.

(ii) Let $N$ be a normal subgroup of $G$. Suppose $G$ has r.x.e, $N$ has r.x.1 and $[G: N]=p^{n}$. Then $G$ has a normal abelian subgroup $A$ with [G:A] dividing $p^{n+2}$.

Proof. We consider (i) first. By Theorem $C$ of [5] we can assume that $N$ has a normal abelian subgroup $A$ of index $p$. If $A$ is not characteristic then $N$ has another such subgroup $B$. Thus $N=A B$, and since both $A$ and $B$ are abelian, $A \cap B \subseteq 3(N)$. Since $[N: A \cap B]=p^{2}$, this results follows.

If $N$ in (ii) has a characteristic abelian subgroup $A$ of index dividing $p^{2}$, the result is clear. Otherwise by (i), $[N: 3(N)]<p^{3}$. Now $3(N) \Delta G$ and $G / 3(N)$ is a $p$-group. Let $A$ be the inverse image in $G$ of a central subgroup of order $p$ of this quotient. Then clearly $A$ is

Received December 26, 1964. The material included here comprises the third chapter of the author's doctoral dissertation written at Harvard University. 
a normal abelian subgroup of $G$ of index dividing $p^{n+2}$.

We will have need for some known results, which we tabulate below.

(1.2) Lemma. Let $G$ be an arbitrary group.

(i) Let $B$ be a normal abelian subgroup of $G$ such that $G / B$ is a p-group. Then $B$ can be extended to $A$, a normal self-centralizing abelian subgroup of $G$.

(ii) Let $N$ be a nontrivial normal subgroup of p-group $G$. Then $N \cap 3(G)>1$.

(iii) Let $K$ and $N$ be subgroups of $G$ with $(K, M) \subseteq 3(G)$. For any $k \in K$ the map $m \rightarrow(k, m)$ is a homomorphism of $M$ into $3(G)$. For any $m \in M$ the map $k \rightarrow(k, m)$ is also a homomorphism. In particular, if $K=G$ and $M=乃_{2}(G)$, the second center of $G$, then the result holds and hence $\left(G^{\prime}, 3_{2}(G)\right)=1$.

(iv) Let $x$ be an element of order $p$ acting on an abelian $p^{\prime-}$ subgroup $H$ of $G$. Then $x$ acts fixed point free on $H / \mathfrak{S}_{H}(x)$.

Proof. (i) If $\subseteq(B)>B$ then since $B$ is normal so is $\subseteq(B)$ and we can join them by a principal series of $p$-group $G / B$. Thus we can find subgroup $C$ normal in $G$ with $\quad \subseteq(B) \supseteqq C>B$ and $[C: B]=p$. Clearly $C$ is abelian and we continue this process.

(ii) Since $G$ is nilpotent, $N$ contains $V$ a subgroup of order $p$ normal in $G$. $G$ acts by conjugation on $V$, a group with automorphism group of order $p-1$. Hence $G$ fixes $V$ and $V \subseteq 3(G)$.

(iii) For the first case we use the commutator identity ([2], p. 150) $(u, v w)=(u, w)(u, v)^{w}$ to obtain

$$
\left(k, m_{1} m_{2}\right)=\left(k, m_{2}\right)\left(k, m_{1}\right)^{m_{2}}=\left(k, m_{1}\right)\left(k, m_{2}\right)
$$

since $\left(k, m_{1}\right)$ is central. For the second case we use $(u v, w)=(u, w)^{v}(v, w)$ to conclude

$$
\left(k_{1} k_{2}, m\right)=\left(k_{1}, m\right)^{k_{2}}\left(k_{2}, m\right)=\left(k_{1}, m\right)\left(k_{2}, m\right)
$$

since $\left(k_{1}, m\right)$ is central.

(iv) Suppose $x$ does not act fixed point free on $H / \mathfrak{S}_{H}(x)$. Set $H_{1}=\mathfrak{\complement}_{H}(x)$ and let $H_{2}$ be the complete inverse image in $H$ of $\mathfrak{\complement}_{H / H_{1}}(x)$. Then $x$ acts on $H_{2}$. Set $G_{1}=\left\langle x, H_{2}\right\rangle$. Clearly $H_{1}=3\left(G_{1}\right)$ since $\left.H_{2}\right\rangle H_{1}$. Also $G_{1} / H_{1}$ is abelian, so $G_{1}^{\prime} \subseteq H_{1}=3\left(G_{1}\right)$. Hence $G_{1}$ is nilpotent of class 2 and so the $p$ - and $p^{\prime}$-elements commute. Thus $x$ centralizes $H_{2}$, a contradiction.

(1.3) Lemma. Let $G$ be a group of order $p^{4}$. Then $G$ has a normal, abelian subgroup $A$ of index $p$. 
Proof. If $G$ is abelian the result is obvious. Suppose $|3(G)|=p^{2}$. Then we need only take $A$ to be a subgroup of index $p$ containing $3(G)$. Thus we can assume $|3(G)|=p$. Let $\zeta \in 3_{2}(G)-3(G)$ with $\zeta^{p} \in 3(G)$. By (iii) of the above lemma $g \rightarrow(g, \zeta)$ is a homomorphism with image $3(G)$ and kernel $\subseteq(\zeta)$. Hence $[G: \subseteq(\zeta)]=p$. But $[\mathfrak{G}(\zeta):\langle\zeta, 3(G)\rangle]=p$, so $\mathfrak{\complement}(\zeta)$ is abelian and the result follows.

(1.4) Lemma. Let $G$ be a group of order $p^{6}$ which is not elementary abelian. Then we can find $g \in B(G)$ and $h \in G^{\prime}$ so that $\langle g, h\rangle$ is a normal subgroup of order $p^{2}$.

Proof. If $3(G)$ contains an element of order $p^{2}$ we can take this for $g$ and $h=1$. So we can assume that $3(G)$ has period $p$ and also that $G$ is nonabelian. We show now that $G$ has a central subgroup $N$ of order $p$ with $N \neq G^{\prime}$. This is clear if $|3(G)|>p$. If $|3(G)|=p$ and $G^{\prime}=3(G)$, then $\left|G^{\prime}\right|=p$ and $p^{5}=[G: 3(G)]=p^{2 e}$ by Lemma 2.3 of [5], a contradiction. Thus we can find such an $N$ with $N<N G^{\prime}$ and both groups are normal. Choose $L$ normal in $G$ with $|L|=p^{2}$ and $N<L \subseteq N G^{\prime}$. The generators of $L$, one from $N$ and the other from $G^{\prime}$, are the required elements.

2. Groups with nontrivial kernel. For convenience we make the following definition.

(2.1) Definition. We say $e(G)=e$ if $G$ has r.x.e but not r.x. $(e-1)$. If $e(G)=2$ we set $\Omega(G)=\cap \operatorname{ker} \theta$ where $\theta$ runs over all irreducible characters of $G$ of degree $p^{2}$.

In this section we characterize those groups $G$ in which the kernel $\Omega(G)$ is nontrivial.

(2.2) Proposition. Let $e(G)=2$ and suppose $\Omega(G)>1$. Then $p==2$ and $G$ has a normal subgroup $N$ of index 2 such that $\Omega(G), N^{\prime}$ and $(G, 3(N))$ are the three distinct subgroups of order 2 of a central subgroup $U$ of type $(2,2)$. Moreover, we have $[G: 3(G)]=2^{4}$.

Conversely, let $[G: 3(G)]=2^{4}$ and let $G$ have a normal subgroup $N$ of index 2 such that $N^{\prime}$ and $(G, 3(N))$ are distinct central subgroups of order 2. Then for $p=2$ we have $e(G)=2$ and $\Omega(G)$ is the third subgroup of order 2 in $\left\langle N^{\prime},(G, 3(N))\right\rangle=U$.

Proof of converse. Since $N$ is a maximal subgroup of $G$ and $3(N)$ is not central in $G$ we must have $N=\mathbb{E} 3(N)$, the centralizer of the center of $N$. Thus $3(G) \subseteq N$ and hence $3(G)<3(N)$. Now $N$ is nonabelian so $[N: 3(N)]=2^{2}$. We show now that for $p=2, e(G)=2$. Clearly $e(G)=1$ or 2 by Propositions 1.1 and 1.4 of [5] since $[G: 3(G)]=2^{4}$. Hence it suffices to show that $G$ does not have r.x.1, 
We suppose $G$ has r.x.1. By Lemma 1.1 (i), $G$ has a normal abelian subgroup $A$ of index 2. Clearly $A \neq N$ so $B=A \cap N$ is a normal abelian subgroup of $N$ of index 2 . Since $N$ is nonabelian $\widetilde{\varsigma}_{N}(B)=B$. Hence $B>3(N)$. Thus $\sqrt{5} 3(N) \supseteqq\langle N, A\rangle=G$, a contradiction, and we have $e(G)=2$.

Let $\theta$ be a character of $G$ of degree $2^{2}$ and let $\theta \mid U=2^{2} \lambda$. Since $N$ cannot be abelian in the representation associated with $\theta$ we see that $N^{\prime} \nsubseteq \operatorname{ker} \lambda$. Similarly $3(N)$ is not central in the representation since $[G: 3(N)]=2^{3}$ so $(G, 3(N)) \nsubseteq$ ker $\lambda$. Hence, since $U$ has only three subgroups of order 2 , the third subgroup must be contained in the kernel of $\lambda$ and hence in the kernel of $\theta$. Since this is true for all such $\theta$, we see that this third subgroup is contained in $\Omega(G)$. We will prove in the forward direction of this proposition that $|\Omega(G)| \leqq 2$. Therefore, with this additional fact, the result follows.

(2.3) Lemma. Let $G$ be an arbitrary group.

(i) Let $g$ and $h$ be two nonidentity elements of $G$. Then there is an irreducible character $\chi$ of $G$ with $g, h \in \operatorname{ker} \chi$.

(ii) Let $G_{1}, G_{2}$ and $G_{3}$ be three nonidentity subgroups of $G$. Suppose that for every irreducible character $\chi$ of $G$ we have $G_{i} \subseteq \operatorname{ker} \chi$ for some $i=1,2$, or 3 . Then the $G_{i}$ are the three distinct subgroups of order 2 of an abelian subgroup $U$ of $G$ of type $(2,2)$.

(iii) Let $G_{1}, G_{2}, G_{3}$ and $G_{4}$ be four nonidentity subgroups of $G$. Suppose that for every irreducible character $\chi$ of $G$ we have $G_{i} \leqq \operatorname{ker} \chi$ for some $i=1,2,3$ or 4 . Then at most two of the $G_{i}$ can have order $\geqq 4$.

Proof. Let $C[G]$ denote the group algebra of $G$ over the complex numbers $\boldsymbol{C}$. We use the fact that this algebra is semi-simple. Thus, if in the algebra an expression of the form $\Pi_{i}\left(1-g_{i}\right)$ is not zero, then we can find an irreducible representation $\mathscr{E}$ with $\mathscr{E}\left(\Pi_{i}\left(1-g_{i}\right)\right) \neq 0$ and hence $\mathscr{X}\left(g_{i}\right) \neq 1$.

(i) It suffices to show here that $(1-g)(1-h) \neq 0$. But if $(1-g)(1-h)=0$ we have

$$
1+g h=g+h .
$$

Since 1 occurs on the left, it occurs on the right, a contradiction since $g \neq 1$ and $h \neq 1$.

(ii) Let $g_{i} \in G_{i}$ with $g_{i} \neq 1$. Then for all such choices we must have $\left(1-g_{1}\right)\left(1-g_{2}\right)\left(1-g_{3}\right)=0$, or

$$
1+g_{1} g_{2}+g_{1} g_{3}+g_{2} g_{3}=g_{1}+g_{2}+g_{3}+g_{1} g_{2} g_{3} .
$$

Since 1 occurs on the left, it occurs on the right. But $g_{i} \neq 1$, so 
$1=g_{1} g_{2} g_{3}$. If $g_{1}$ and $g_{2}$ are fixed, then this holds for all $g_{3} \in G_{3}-\{1\}$. Hence $\left|G_{3}\right|=2$ and similarly for $G_{1}$ and $G_{2}$. Also the above equation implies that $G_{1}, G_{2}$ and $G_{3}$ are the three distinct subgroups of $U=$ $\left\langle G_{1}, G_{2}, G_{3}\right\rangle$ which is abelian of type $(2,2)$.

(iii) Suppose first that two of these groups have a nontrivial intersection. Say $G_{3} \cap G_{4}=H>1$. Then applying (ii) above to the groups $G_{1}, G_{2}$ and $H$ we see that $\left|G_{1}\right|=\left|G_{2}\right|=2$, so the result follows here. Hence we can assume that for $i \neq j$ we have $G_{i} \cap G_{j}=1$. Suppose $g \in G$ can be written in the form $g=g_{i} g_{j}$ with $g_{i} \in G_{i}$ and $g_{j} \in G_{j}$. Then for fixed $i$ and $j$ this representation is unique. If not, we can find $\bar{g}_{i} \in G_{i}$ and $\bar{g}_{j} \in G_{j}$ with $g_{i} \neq \bar{g}_{i}$ and $g=g_{i} g_{j}=\bar{g}_{i} \bar{g}_{j}$. Hence

$$
\bar{g}_{i}^{-1} g_{i}=\bar{g}_{j} g_{j}^{-1} \in G_{i} \cap G_{j}==1 \text {. }
$$

So $g_{i}=\bar{g}_{i}, g_{j}=\bar{g}_{j}$, a contradiction.

Let us assume $\left|G_{2}\right| \geqq 3$ and $\left|G_{3}\right| \geqq 4$. We show that $\left|G_{1}\right|=\left|G_{4}\right|=2$. This will clearly yield the result. Choose nonidentity $g_{1} \in G_{1}$. Since $\left|G_{2}\right| \geqq 3$, we can choose nonidentity $g_{2} \in G_{2}$ with $g_{1} \notin g_{2} G_{4}$. This follows by the uniqueness mentioned above. Since $\left|G_{3}\right| \geqq 4$, we can choose nonidentity $g_{3} \in G_{3}$ with $g_{1} \notin g_{3} G_{4}$ and $g_{1} \notin G_{2} g_{3}$. Now for all $g_{4} \in G_{4}-\{1\}$ we have

$$
\left(1-g_{1}^{-1}\right)\left(1-g_{2}\right)\left(1-g_{3}\right)\left(1-g_{4}^{-1}\right)=0
$$

or

$$
\begin{aligned}
1 & +g_{1}^{-1} g_{2}+g_{1}^{-1} g_{3}+g_{1}^{-1} g_{4}^{-1}+g_{2} g_{3}+g_{2} g_{4}^{-1}+g_{3} g_{4}^{-1}+g_{1}^{-1} g_{2} g_{3} g_{4}^{-1} \\
& =g_{1}^{-1}+g_{2}+g_{3}+g_{4}^{-1}+g_{1}^{-1} g_{2} g_{3}+g_{1}^{-1} g_{2} g_{4}^{-1}+g_{1}^{-1} g_{3} g_{4}^{-1}+g_{2} g_{3} g_{4}^{-1}
\end{aligned} .
$$

Since 1 appears on the left side, it must appear on the right. But $g_{i} \neq 1$ and $g_{1} \notin G_{2} g_{3}, g_{2} G_{4}$ or $g_{3} G_{4}$. Hence we must have $1=g_{2} g_{3} g_{4}^{-1}$ or $g_{4}=g_{2} g_{3}$. Since this is true for all nonidentity $g_{4} \in G_{4}$, we have $\left|G_{4}\right|=2$. Finally interchanging the roles of $G_{4}$ and $G_{1}$ yields $\left|G_{1}\right|=2$ and the result follows.

(2.4) Lemma. Let $e(G)=2$. Let $N$ be a normal subgroup of $G$. Then

(i) if $e(N)=2$ then $\Omega(G) \cap N \subseteq \Omega(N)$,

(ii) if $e(G / N)=2$ then $\Omega(G) N / N \subseteq \Omega(G / N)$,

(iii) $\Omega(G) \subseteq G^{\prime}$.

Proof. Let $x \in \Omega(G) \cap N$ with $x \neq 1$. If $x \notin \Omega(N)$ we can choose character $\varphi$ of $N$ of degree $p^{2}$ with $x \notin \operatorname{ker} \varphi$. Let $\chi$ be a constituent of $\varphi^{*}$. Then $p^{2}=\operatorname{deg} \varphi \leqq \operatorname{deg} \chi \leqq p^{2}$, so $\operatorname{deg} \chi=\operatorname{deg} \varphi$ and $\chi \mid N=\varphi$. Hence $x \notin \operatorname{ker} \chi$ and $\operatorname{deg} \chi=p^{2}$, a contradiction. Thus $x \in \Omega(N)$ and (i) follows.

Part (ii) follows easily, since any character of $G / N$ of degree $p^{2}$ 
can be viewed in a natural way as one of $G$.

Let $x$ be a nonidentity element of $\Omega(G)$ and let $\theta$ be a character of $G$ of degree $p^{2}$. We have $x \in \operatorname{ker} \theta$. If $x \notin G^{\prime}$ then we can find a linear character $\lambda$ of $G$ with $\lambda(x) \neq 1$. This implies that $\theta \lambda$ is an irreducible character of $G$ of degree $p^{2}$ with $x \notin \operatorname{ker} \theta \lambda$, a contradiction. This completes the proof.

(2.5) Lemma. Let $e(G)=2$ and $\Omega(G)>1$. Suppose also that $G$ has a normal subgroup $N$ of index $p$ with $\sqrt{5} 3(N)=N$. Then $p=2$, $|\Omega(G)|=2,[G: 3(G)]=2^{4}$ and $\Omega(G), N^{\prime}$ and $(G, 3(N))$ are the three distinct subgroups of order 2 of a central subgroup $U$ of type (2.2).

Proof. Since $\Omega(G) \subseteq G^{\prime}$ we see that $\Omega(G) \subseteq N$. Set $G_{1}=\Omega(G)$, $G_{2}=(G, 3(N))$ and $G_{3}=N^{\prime}$. By assumption, $G_{2}>1$ and since $e(G)=2$, $N$ is nonabelian and $G_{3}>1$.

Suppose $G$ has a character $\chi$ which does not contain either $G_{1}, G_{2}$ or $G_{3}$ wholly in its kernel. Since $\Omega(G)=G_{1}$ is not in the kernel and $\Omega(G) \subseteq G^{\prime}$, we see that $\operatorname{deg} \chi=p$. We consider $\chi \mid N$. If $\chi \mid N=\varphi$ is irreducible, then clearly $3(N)$, being central in the representation associated with $\varphi$, is in the center of $\chi$. But this implies that $(G, 3(N))=G_{2} \subseteq$ ker $\chi$, which is not the case. On the other hand, suppose $\chi \mid N=\lambda_{1}+\lambda_{2}+\cdots+\lambda_{p}$, a sum of $p$ linear characters. Then clearly $N^{\prime}=G_{3} \subseteq$ ker $\chi$, again a contradiction. Thus no such $\chi$ exists and by Lemma 2.3 (ii) we see that $G_{1}, G_{2}$ and $G_{3}$ are the three distinct subgroups of order 2 of $U=\left\langle G_{1}, G_{2}, G_{3}\right\rangle$ which is abelian of type $(2,2)$. Since the $G_{i}$ are clearly normal subgroups of $G$, they are central since they have order 2 , and thus $U$ is central. In particular $3(N) \supseteqq N^{\prime}$, so $N$ is nilpotent of class 2. Since $\mathfrak{g}(N)$ is abelian and central in $N$, this implies that $N^{\prime}$ is a $p$-group. Hence $p=2$.

If $e(N)=2$, then $\Omega(G)=\Omega(G) \cap N \subseteq \Omega(N) \subseteq N^{\prime}$ and so $G_{1}=G_{3}$, which is not the case. Since $N$ is nonabelian we must have $e(N)=1$. By Lemma 2.3 of [5], $[N: 3(N)]=2^{2}$. Now $G / N$ acts on $3(N)$ and $|(G, 3(N))|=2$. Thus applying Lemma 1.2 (iii), since $3(N) \supseteqq 3(G)$, we have $[3(N): 3(G)]=2$ and hence $[G: 3(G)]=2^{4}$. This completes the proof.

Proof of Proposition 2.2. By induction on $|G|$. If $G$ has a normal subgroup $N$ of index $p$ with $53(N)=N$, then the result follows by the previous lemma. Hence we assume that for all such $N, 3(N) \subseteq$ $3(G)$ and we obtain a contradiction.

We first show that $G$ is nilpotent. Let $N$ be a normal subgroup of $G$ of index $p$ (see Proposition 3.4 of [5]). If $e(N)=2$ then $\Omega(G) \subseteq G^{\prime} \subseteq N$ so $\Omega(N)>1$. Hence $p=2$ and $[N: 3(N)]=2^{4}$ by induction. Thus $[G: 3(G)]=2^{4}$ or $2^{5}$. Now suppose $N$ has $r_{0}, x, 1$. Clearly 
$N$ is nonabelian. If $[N: 3(N)]=p^{2}$ or $p^{3}$, then $[G: 3(G)]=p^{3}$ or $p^{4}$. Hence in either case, $G$ is nilpotent. The remaining possibility is that $N$ has a characteristic abelian subgroup $A$ of index $p$ (Lemma 1.1 (i)), and so $G$ has a normal abelian subgroup $A$ of index $p^{2}$.

We study this latter case. We show first that $G / A$ is abelian of type $(p, p)$. Suppose not. Then $G / A$ is cyclic, and so there is a unique subgroup $N$ of $G$ with $G>N>A$. Clearly $N$ is nonabelian. By Lemma 2.3 (i), $G$ has an irreducible character $\chi$ not containing either $N^{\prime}$ or $\Omega(G)$ wholly in its kernel. Since $\Omega(G) \nsubseteq \operatorname{ker} \chi$, we see that $\operatorname{deg} \chi=p$. Also $N^{\prime} \nsubseteq \operatorname{ker} \chi$ implies $\chi \mid N=\varphi$ is irreducible. Now $A$ is abelian, so $\chi|A=\varphi| A=\lambda_{1}+\lambda_{2}+\cdots+\lambda_{p}$, a sum of $p$ distinct conjugates since, if $\varphi \mid A=a \sum_{1}^{t} \lambda_{i}$, we have $a^{2} t \leqq[N: A]=p$. Let $T$ be the inertia group of $\lambda=\lambda_{1}$. Then $G>T>A$, so $T=N$. But this implies that $\lambda_{1}$ is fixed by $N$, contradicting $\varphi \mid A=\lambda_{1}+\cdots+\lambda_{p}$. Thus $G / A$ is abelian of type $(p, p)$.

Write $A=H \times P$ where $P=\mathfrak{S}_{p}(A)$ and $H$ is the Hall $p^{\prime}$-subgroup of $G$. Both are clearly normal in $G$. We show that $H$ is central. If not, let $H_{1}=H \cap 3(G)<H$. Let $g$ be an element of order $p$ of $G / A$. Now $G / A$ acts on $H$, since $A$ is abelian. Clearly $\widetilde{E}_{H}(g) \supseteqq H_{1}$. On the other hand $\mathfrak{S}_{H}(g) \subseteq 3(\langle A, g\rangle) \subseteq 3(G)$ by assumption, since $\langle A, g\rangle$ has index $p$ in $G$. Hence $H_{1}=\mathfrak{S}_{H}(g)$. By Lemma 1.2 (iv), $g$ acts fixed point free on $H / H_{1}$ and this is true for all $g$. Then group $G / A$, which is abelian of type $(p, p)$, acts fixed point free on $H / H_{1}$, a contradiction ([1], Theorem V, p. 336). Hence $H$ is central and $G$ is nilpotent.

We have $G=\mathfrak{g}(G) \times \mathfrak{S}_{p}(G)$. Let $\zeta \in \mathrm{Z}_{2}(G)-3(G)$ with $\zeta^{p} \in 3(G)$. For any $g \in G$ we have $(g, \zeta) \in 3(G)$ and $(g, \zeta)^{p}=\left(g, \zeta^{p}\right)=1$. Hence $(G, \zeta)$ is central and has period $p$. If $|(G, \zeta)|=p$, then $[G:[(\zeta)]=p$ and $\zeta \in 3 \subseteq(\zeta) \subseteq 3(G)$, a contradiction. Hence $|(G, \zeta)| \geqq p^{2}$. Thus we can choose a central subgroup $J$ of order $p$ with $J \nsupseteq \Omega(G)$. Consider $\bar{G}=G / J$. We show first that $e(\bar{G})=2$.

Since $\Omega(G) \subseteq G^{\prime}$ we see that $\bar{G}$ is nonabelian. If $e(\bar{G})=1$ then either $\bar{G}$ has an abelian subgroup $\bar{B}$ of index $p$ or $[\bar{G}: 3(\bar{G})]=p^{3}$. Suppose the first case occurs. Let $B$ be the complete inverse image of $\bar{B}$ in $G$. Clearly $B$ is nonabelian. If $e(B)=2$ then $\Omega(G) \leqq B^{\prime}=J$, a contradiction. Hence $e(B)=1$ and $[B: 3(B)]=p^{2}$ by Lemma 2.3 of [5]. Thus $[G: 3(G)]=p^{2}$ or $p^{3}$, which contradicts $e(G)=2$. On the other hand, suppose $[\bar{G}: 3(\bar{G})]=p^{3}$. Let $\zeta$ belong to the complete inverse image of $3(\bar{G})$ in $G$. Then $(G, \zeta) \subseteq J$ so $[G$ : $\subseteq(\zeta)]=1$ or $p$. Hence $\zeta \in \mathbb{Z} \subseteq(\zeta) \subseteq 3(G)$ and $[G: B(G)]=p^{2}$ or $p^{3}$, again a contradiction. Thus $e(\bar{G})=2$.

By Lemma 2.4 (ii), $\Omega(\bar{G})>1$. Hence by induction $p=2,[\bar{G}: 3(\bar{G})]=2^{4}$ and $\bar{G}$ has a normal subgroup $\bar{N}$ of index 2 with $[\bar{N}: 3(\bar{N})]=2^{2}$. As in the above, $[\bar{G}: 3(\bar{G})]=2^{4}$ implies that $[G: 3(G)]=2^{4}$. Let $D$ be the complete inverse image in $G$ of $3(\bar{N})$. Since $[D: 3(G)]=2$, we can write $D=\langle 3(G)$, $\eta\rangle$ with $\eta \in Z_{2}(G)$. Now $|(\bar{G}, 3(\bar{N}))|=2$ and $|J|=$ 
$p=2$ so $|(G, \eta)|=2$ or 4 . If $|(G, \eta)| \leqq 2$ then $\eta$ would be central, a contradiction. Hence $|(G, \eta)|=4$ and $[G: \subseteq(\eta)]=4$. This means that $[\mathfrak{\Im}(\eta):\langle 3(G), \eta\rangle]=2$ and hence $A=\mathbb{}(\eta)$ is a normal abelian subgroup of $G$. Moreover, since $\eta^{2} \in 3(G),(G, \eta)$ has period 2. Hence $G / A \simeq(G, \eta)$ is abelian of type $(2,2)$.

Let us denote by $N_{1}, N_{2}$ and $N_{3}$ the three subgroups of $G$ satisfying $G>N_{i}>A$. Since clearly $A=\mathfrak{C}(A) \supseteqq 尺(G)$ we have $N_{i} \supseteqq 3(G)$ and hence $\left[N_{i}: 3\left(N_{i}\right)\right]=\left[N_{i}: 3(G)\right]=2^{3}$. Each $N_{i}$ is nonabelian and has a normal abelian subgroup of index 2 . Hence, by considering the action of $N_{i} / A$ on $A$, we obtain $\left|N_{i}^{\prime}\right|\left|3\left(N_{i}\right)\right|=|A|$ and so $\left|N_{i}^{\prime}\right|=4$. Set $G_{i}=N_{i}^{\prime}$ for $i=1,2,3$ and set $G_{4}=\Omega(G)$. Since $\left|G_{1}\right|=\left|G_{2}\right|=\left|G_{3}\right|=4$ and $\left|G_{4}\right|>1$, we see by Lemma 2.3 (iii) that there is an irreducible character $\chi$ of $G$ with $G_{i} \nsubseteq \operatorname{ker} \chi$ for $i=1,2,3,4$.

Since $\Omega(G) \nsubseteq \operatorname{ker} \chi$ and $\Omega(G) \subseteq G^{\prime}$ we see that $\operatorname{deg} \chi=2$. We consider $\chi \mid A$. Since $A$ is abelian, we have either $\chi \mid A=2 \lambda$ or $\chi \mid A=$ $\lambda_{1}+\lambda_{2}$. In the first case $A$ would be central in the representation. This would imply that each $N_{i}$ is abelian in the representation and hence $G_{i}=N_{i}^{\prime} \leqq \operatorname{ker} \chi$ for $i=1,2,3$, which is not the case. Thus $\chi \mid A=$ $\lambda_{1}+\lambda_{2}$. The inertia group $T$ of $\lambda_{1}$ satisfies $G>T>A$ so $T$ is one of the $N_{i}$, say $N_{1}$. Suppose $\chi \mid N_{1}=\varphi$ is irreducible. Then $\varphi \mid A=\lambda_{1}+\lambda_{:}$, which is not the case, since $\lambda_{1}$ and $\lambda_{2}$ are not conjugate in $T=N_{1}$. Hence $\chi \mid N_{1}$ has only linear constituents and so $G_{1}=N_{1}^{\prime} \leqq \operatorname{ker} \chi$, again a contradiction. This completes the proof.

We show by example now that such groups exist.

(2.6) ExAmple. Let $D$ be the dihedral group of order 8 and let $D^{*}=D \times D$. Now for $p=2, e(D)=1$ and in fact $D$ has precisely one nonlinear character. Then clearly $D^{*}$ has $e\left(D^{*}\right)=2$ and precisely one character of degree $2^{2}$. But $D^{*}$ is not a faithful linear group, since its center is not cyclic, so $\Omega\left(D^{*}\right)>1$.

The reason for studying groups $G$ with $\Omega(G)>1$ can be seen from the following.

(2.7) Proposition. Let $G$ have r.x.2 and suppose that $G$ has a normal subgroup $N$ of index $p$ with $e(N)=2$ and $N=53(N)$. Then $p=2,(G, 3(N))=\Omega(N)>1$ and $[G: 3(G)]=2^{6}$.

Conversely, let $G$ have a subgroup $N$ of index 2 with $e(N)=2$ and $\Omega(N)>1$. Suppose also that $(G, 3(N))=\Omega(N)$. Then for $p=2$ we have $e(G)=2$.

Note that, using the structure of groups $N$ with $\Omega(N)>1$ as given in Proposition 2.2, the structure of group $G$ above is as follows. $G$ has subgroups $N$ and $K$ with 


$$
G>\underbrace{}_{2} N \underset{2}{L_{2}} K \underbrace{>}_{2^{2}} 3(K)>\underbrace{>}_{2} 3(N)>\underbrace{}_{2} 3(G)>1
$$

and $(G, 3(N)),(N, 3(K))$ and $K^{\prime}$ are the three distinct subgroups of order 2 of $U$, an abelian subgroup of type $(2,2)$ contained in $3(N)$.

Proof. We show first that $\Omega(N) \supseteqq(G, 3(N))>1$. Let $x$ be a nonidentity element of $(G, 3(N))$. If $x \notin \Omega(N)$, then there exists a character $\varphi$ of $N$ of degree $p^{2}$ with $x \notin \operatorname{ker} \varphi$. Let $\chi$ be a constituent of $\phi^{*}$. Then, since $e(G)=2$, we have $p^{2} \geqq \operatorname{deg} \chi \geqq \operatorname{deg} \varphi=p^{2}$. Hence $\operatorname{deg} \chi=\operatorname{deg} \varphi$ and $\chi \mid N=\varphi$. Thus $3(N)$ being central in the representation associated with $\phi$ is central in the representation associated with $\chi$. But this implies that $(G, 3(N)) \subset \operatorname{ker} \chi \cap N=\operatorname{ker} \varphi$, which is not the case. Thus $\Omega(N) \supseteqq(G, 3(N))>1$ and Proposition 2.2 applies here.

Clearly $N \supseteqq 3(G)$, so $3(N)>3(G)$. Now we have $p=2$ and $[N: 3(N)]=2^{4}$. Moreover, $|\Omega(N)|=2$ and so $|(G, 3(N))|=2$. Let $G=\langle N, \eta\rangle$. Then $3(G)=\subseteq(\eta) \cap 3(N)$ is the kernel of the homomorphism $a \rightarrow(\eta, a)$ of $3(N)$ into $(G, 3(N))$. Hence $[3(N): 3(G)]=2$ and $[G: 3(G)]=2^{6}$. This completes the forward direction of the proof.

Conversely we see as above that $|(G, 3(N))|=2$ implies that $[3(N): 3(G)]=2$ and hence, since $[N: 3(N)]=2^{4}$ by Proposition 2.2 , we have $[G: 3(G)]=2^{6}$. For $p=2$, Propositions 1.1 and 1.4 of [5] imply that $G$ has r.x.3. Hence we need only show that $G$ has no irreducible character of degree $2^{3}$. Suppose $\chi$ were such a character. Then since $N$ has r.x.2 we must have $\chi \mid N=\varphi_{1}+\varphi_{2}$, the sum of two conjugate characters of $N$ of degree $2^{2}$. But then $\Omega(N) \cong \operatorname{ker} \varphi_{1} \cap \operatorname{ker} \varphi_{2}$, so $\Omega(N)=(G, 3(N)) \subseteq \operatorname{ker} \chi$. This implies that $3(N)$ is central in the representation associated with $\chi$, a contradiction, since $[G: 3(N)]=2^{5}$.

Finally we give an example of such a group.

(2.8) Example. Let $D^{*}=D \times D$ be the group given in Example 2.6. $D^{*}$ has an obvious automorphism of degree 2 which amounts to essentially interchanging the two factors. Let $F_{3}$ be the semidirect product of $D^{*}$ by an element of order 2 acting in this manner.

Now we have shown for $p=2$ that $e\left(D^{*}\right)=2$ and $\Omega\left(D^{*}\right)>1$. Moreover, it is easy to see that $\left(F_{3}, 3\left(D^{*}\right)\right)=\Omega\left(D^{*}\right)$. Hence by the above Proposition, $F_{3}$ has r.x.2.

3. Self-centralizing abelian subgroups, We saw in the last section that the prime 2 plays a special role in the characterization of groups with r.x.2. We discuss now another special case which can occur. 
(3.1) ExAmple. Let $G_{0}$ be the following group of order $72 . G_{0}$ is the semidirect product of $V_{0}$, an abelian group of type $(3,3)$ by $D_{0}$, the dihedral group of order 8. We write this as $G_{0}=V_{0} \times{ }_{\sigma} D_{0}$. The action of $D_{0}$ on $V_{0}$ is a faithful irreducible representation of degree 2 of $D_{0}$ over $G F(3)$. Now $D_{0}$ has only one nonlinear irreducible representation and this is realizable over $G F(3)$, so $G_{0}$ is well defined by this information.

Let us consider a concrete example of the representation. $D_{0}$ is generated by elements $x$ and $y$ of order 4 and 2 respectively, given by

$$
x=\left[\begin{array}{cc}
0 & 1 \\
-1 & 0
\end{array}\right] \quad y=\left[\begin{array}{cc}
1 & 0 \\
0 & -1
\end{array}\right]
$$

Now $D_{0}$ has four noncentral elements of order 2 . Let $w$ be such an element. Since $w+1 \neq 0, w-1 \neq 0$ and $0=w^{2}-1=(w+1)(w-1)$, we see that $(w-1) V_{0}$ is a one-dimensional subspace of $V_{0}$ and this is invariant under $w$. If $(w-1) V_{0}=(\bar{w}-1) V_{0}$ for some other noncentral element $\bar{w}$ of order 2 , then clearly

$$
(\bar{w}+1)(w-1)=(\bar{w}-1)(w+1)=0,
$$

so $w=\bar{w}$, a contradiction. Hence the four subspaces $(w-1) V_{0}$ for the four elements of order 2 are all distinct. But $V_{0}$ has only four subspaces of dimension 1 and thus every one is of this form.

Since $V_{0}$ is a normal abelian subgroup of $G_{0}$ we have by Proposition 1.1 of [5] that $G_{0}$ has r.x.3 for $p=2$. We show now that $G_{0}$ has r.x.2.

Let $\lambda$ be a nonprincipal linear character of $V_{0}$. Then $\operatorname{ker} \lambda$ is a one-dimensional subspace of $V_{0}$ and so $\operatorname{ker} \lambda=\left(w, V_{0}\right)$ by the above in multiplicative language for some noncentral element $w$ of $D_{0}$ of order 2. Then

$$
\lambda^{w}(v)=\lambda\left(w v w^{-1}\right)=\lambda\left(w v w^{-1} v^{-1}\right) \lambda(v)=\lambda(v)
$$

and so $w$ fixes $\lambda$. Thus $\lambda$ has 1,2 or 4 conjugates. In the first two cases we would have $\lambda$ fixed by $z$, the central element of $D_{0}$. But $z v z^{-1}=v^{-1}$ so $\lambda^{z}(v)=\lambda\left(v^{-1}\right)=\lambda(v)$ implies $\lambda\left(v^{2}\right)=1$, which is not the case for all $v$. Hence $\lambda$ has 4 conjugates.

Now let $\chi$ be a character of $G_{0}$ with $\chi \mid V_{0}=a \sum_{1}^{t} \lambda_{i}$. If $t=1$, then $\lambda=\lambda_{1}$ is the principal character of $V_{0}$ and hence $\chi$ is a character of $D_{0}$ and has degree 1 or 2 . If $t \neq 1$, then $\lambda=\lambda_{1}$ is a nonprincipal character and hence $t=4$. Since $a^{2} t \leqq\left[G_{0}: V_{0}\right]=8$ by Lemma 1.2 of [5], we have $a=1$ and $\operatorname{deg} \chi=4$. Thus $G$ has r.x.2.

For convenience we introduce the following. 
of $G$ of index $p$ we have $3(N) \subseteq 3(G)$.

The main result of this section is the following.

(3.3) Proposition. Let $G$ satisfy Hypothesis 3.2. Moreover, suppose $G$ has a self-centralizing normal abelian subgroup of index $p^{3}$. Then either $[G: 3(G)]$ divides $p^{6}$ or $G$ is a central extension of an abelian group $Z$ by $G_{0}$ (of Example 3.1) such that $Z V_{0}$ is abelian.

Conversely, let $G$ be a group with $[G: 3(G)]$ dividing $p^{5}$ or let $G$ be a central extension of abelian group $Z$ by $G_{0}$ such that $Z V_{0}$ is abelian, then $G$ has r.x.2. In the latter case we of course have $p=2$.

Proof of the converse. If $[G: 3(G)]$ divides $p^{5}$, then the result follows by Propositions 1.1 and 1.4 of [5]. So we assume $G$ has the second structure.

Let $\chi$ be a character of $G$. Say $\chi \mid A=a \sum_{1}^{t} \lambda_{i}$ and $\chi \mid Z=b \ell$ where $A=Z V_{0}$ is abelian. Now the $\lambda_{i}$ are all constituents of $\mu^{*}$ (induction to $A$ ), a character of degree 9 . Since $D_{v}$ fixes $\mu$, we see that $D_{0}$ permutes the constituents of $\mu^{*}$, which are all linear. Since $D_{0}$ is a 2-group, it must fix some constituent, say $\lambda$. Then the characters $\lambda_{i} \bar{\lambda}$ are a full set of conjugate characters of $A / Z=V_{0}$. Hence, by the results proved in Example 3.1, we have either $t=1$ or 4 . Since $a^{2} t \leqq[G: A]=8$ and $a$ is a power of 2 , we see that $a=1$ or 2 if $t=1$ and $a=1$ if $t=4$. Thus $\operatorname{deg} \chi=a t=1,2$ or 4 and the result follows.

(3.4) Lemma. Let $K$ be a proper normal subgroup of $L$.

(i) Let $e(K)=1$ and suppose $L$ fixes all nonlinear characters of $K$. Then $3(K) \cong 3(L)$.

(ii) Let $L$ have r.x.1 and suppose $K$ is nonabelian. Then $3(K) \subseteq 3(L)$.

(iii) Suppose $L$ is a subgroup of $G$, a group with r.x.2. If $K$ is nonabelian and $\sqrt{5} 3(K)=K$, then $e(G)=e(L)=2$.

Proof. Let $e(K)=1$. We show first that for all nonidentity elements $x \in K$ we can find an irreducible nonlinear character $\varphi$ with $x \notin \operatorname{ker} \varphi$. Using the semisimplicity of the group algebra, we can find a character $\lambda$ of $K$ with $x \notin \operatorname{ker} \lambda$. It clearly suffices to assume that $\lambda$ is linear. Let $\theta$ be a character of $K$ of degree $p$. Then clearly either $x \notin \operatorname{ker} \theta$ or $x \notin \operatorname{ker} \theta \lambda$.

Suppose we have (i). We show that $3(K) \subseteq 3(L)$. If not, then $(L, 3(K))$ is a nontrivial subgroup of $K$. Choose nonidentity $x \notin(L, 3(K))$ and let $\varphi$ be a nonlinear character of $K$ with $x \notin \operatorname{ker} \varphi$. Let $\chi$ be a constituent of $\varphi^{*}$. Then $\chi \mid L=a \sum_{1}^{t} \varphi_{i}$ where the $\varphi_{i}$ are the distinct conjugates of $\varphi=\varphi_{i}$. Since $L$ fixes all nonlinear characters of $K$, we have $t=1$ and $\chi \mid L=a \varphi$. Since $3(K)$ is central in the representation 
associated with $\varphi$, it is central in the one associated with $\chi$. Thus $(L, 3(K)) \subseteq \operatorname{ker} \chi \cap K=\operatorname{ker} \varphi$, a contradiction, and (i) follows.

Let $L$ and $K$ be as in (ii). Clearly $e(L)=e(K)=1$. Let $\varphi$ be a nonlinear character of $K$ and let $\chi$ be a constituent of $\phi^{*}$. Then $\chi \mid K=a \sum_{1}^{t} \varphi_{i}$ and $\operatorname{deg} \chi=a t \operatorname{deg} \varphi$. Since $\operatorname{deg} \chi \leqq p$ we have $a=t=1$. Hence $L$ fixes $\varphi$. The result now follows by (i).

Finally, suppose $G, L$ and $K$ are as in (iii). Since $K$ is nonabelian, $e(L) \geqq e(K) \geqq 1$. If $e(L)=1$ then by (ii) above, $L \subseteq \subseteq 3(K)$, which is not the case. Thus $2 \geqq e(G) \geqq e(L) \geqq 2$ and the result is proved.

(3.5) Lemma. Let $G$ satisfy Hypothesis 3.2. Let $K$ be a normal subgroup of $G$ of index $p^{2}$ with $e(K)=1$. If $K=\sqrt{3}(K)$ then $3(K)>3(G)$, and either $[3(K): 3(G)]=p$ or $[G: 3(G)]=p^{6}$. In particular, we see that the Hall $p^{\prime}$-subgroup of $3(K)$ is central.

Proof. $G / K$ is abelian and in fact either type $(p, p)$ or $\left(p^{2}\right)$. We show that the latter cannot occur. Suppose to the contrary that $G / K$ is cyclic. Let $L$ be the unique subgroup with $G>L>K$. Let $\varphi$ be a character of $K$ of degree $p$ and let $\chi$ be a constituent of $\varphi^{*}$. Then $\chi \mid K=a \sum_{1}^{t} \varphi_{i}$. Since $\operatorname{deg} \chi \leqq p^{2}$, we see that $t=1$ or $p$. Then clearly $L$ fixes $\varphi$. Since this is true for all such $\varphi, L \subseteq \mathbb{E} 3(K)$ by Lemma 3.4 (i), a contradiction. Thus $G / K$ is abelian of type $(p, p)$.

Let $H$ be the Hall $p^{\prime}$-subgroup of $3(K)$. This is normal in $G$. We show that it is central. If not, then $H_{1}=H \cap 3(G)<H$. Let $x$ be an element of order $p$ of $G / K$. Now $G / K$ acts on $H$, since $H \subseteq 3(K)$. Clearly, $\mathfrak{\Im}_{H}(x) \supseteqq H_{1}$. On the other hand, $\mathfrak{\Im}_{H}(x) \subseteq 3(\langle K, x\rangle) \subseteq 3(G)$ by Hypothesis 3.2. Hence $H_{1}=\mathfrak{S}_{H}(x)$. By Lemma 1.2 (iv), $x$ acts fixed point free on $H / H_{1}$ and this is true for all $x$. Then noncyclic abelian $p$-group $G / K$ acts fixed point free on $H / H_{1}$, a contradiction ([1] Theorem V, p. 336). Thus $H$ is central.

Let $3(K)=H \times P$ where $P$ is a Sylow $p$-subgroup of $3(K)$. Clearly $P$ is normal in $G$. Also $3(K)>3(G) \supseteqq H$, so $3(G)=H \times P_{1}$ with $P_{1}=P \cap 3(G)<P$. Since $3(K) / 3(G)$ is a normal $p$-subgroup of $G / 3(G)$ we can find an element of order $p$ in the center of a Sylow $p$-subgroup of $G / 3(G)$ in that group by Lemma 1.2 (ii). Let $\zeta$ be an inverse image of this element in $G$ with $\zeta \in P$. Thus $\left(\zeta, \Im_{p}(G)\right) \subseteq 3(G)$ for some Sylow $p$-subgroup of $G$. But $\zeta \in \mathbb{Z}(K)$ and $K \supseteqq \mathfrak{g}(G)$, so $(\zeta, \mathfrak{g}(G))=1$. Hence $\zeta$ is an element of $3_{2}(G)-3(G)$ with $\zeta^{p} \in 3(G)$.

The map $g \rightarrow(g, \zeta)$ is a homomorphism of $G$ into $3(G)$ by Lemma 1.2 (iii). Let $L$ be the kernel. Since $\zeta \in 3(K), L \supseteqq K$. If $L>K$, then $\zeta \in 3(L)=3(G)$ by Hypothesis 3.2. Thus $K=L$ and the group $(G, \zeta) \simeq G / K$ is an elementary abelian group of order $p^{2}$. Let $J$ be a subgroup of $(G, \zeta)$ of order $p$ with $J \nsupseteq K^{\prime}$. Such a choice is clearly possible, since $|(G, \zeta)|=p^{2}$. Under the homomorphism $g \rightarrow(g, \zeta)$ let 
$N$ be the complete inverse image of $J$. Then $N$ is normal and $G>N>K$. Let $M=\langle 3(G), \zeta\rangle$.

Consider the group $\bar{G}=G / J$ with subgroups $\bar{N}, \bar{K}$ and $\bar{M}$. Since $M>3(G) \supseteqq J$, we have

$$
\bar{G}>\bar{N}>\bar{K} \supseteqq 3(\bar{K}) \supseteqq \overline{3(K)} \supseteqq \bar{M}>1 \text {. }
$$

Note that $\bar{G}$ has r.x.2, but that it does not necessarily satisfy $\mathrm{Hy}$ pothesis 3.2. Now $\bar{K}$ is nonabelian by our choice of $J$. There are now two possibilities, either $\sqrt{ } 3(\bar{K})=\bar{K}$ or $(\sqrt{ } 3(\bar{K})>\bar{K}$.

Suppose first $\sqrt{ } 3(\bar{K})=\bar{K}$. Then by Lemma 3.4 (iii) $e(\bar{G})=e(\bar{N})=2$. Now $\bar{M} \subseteq 3(\bar{N})$, since $J=(N, \zeta)$. But $\bar{M} \nsubseteq 3(\bar{G})$, since otherwise $J \supseteqq(G, \zeta)$, which is not the case. Thus $\bar{G}$ satisfies the hypothesis of Proposition 2.7. Hence for $p=2,[\bar{G}: 3(\bar{G})]=p^{6}$. Let $\eta$ be an element of $G$ in the inverse image of $3(\bar{G})$. Then clearly $\eta \in B_{2}(G)$. Now $(G, \eta) \subseteq J$, so by Lemma 1.2 (iii) we see that $[G: \subseteq(\eta)]=1$ or $p$. But by Hypothesis 3.2 we cannot have $[G: \mathfrak{G}(\eta)]=p$. Hence $\eta \in \mathbb{Z}(G)$. Therefore $[G: 3(G)]=p^{6}$.

Now let $\sqrt{ } 3(\bar{K})>\bar{K}$, and in particular $(\overline{(3(\bar{K}))}>\bar{K}$. Choose subgroup $L$ with $G>L>K$ such that $L$ is contained in the complete inverse image of $\sqrt{(3(K)})$. Then $(L, 3(K))=J$. Let $L=\langle K, x\rangle$. The map $b \rightarrow(b, x)$ is then a homomorphism of $B=3(K)$ onto $J$. Thus the kernel which is $3(L)$ has index $p$ in $3(K)$, since $J$ has order $p$. But by Hypothesis $3.2,3(L)=3(G)$ and the result follows.

Let $G$ be a group with r.x.2, and let $A$ be a normal abelian subgroup of index $p^{3}$. Suppose $G / A$ is nonabelian. For each $x \in G$ let $d(x)=\left[A: \mathfrak{S}_{A}(x)\right]$. This is clearly a function of the cosets of $A$. If $n$ is prime to $p$, then clearly $d\left(x^{n}\right)=d(x)$. Also $\mathfrak{F}_{A}\left(x^{y}\right)=\mathfrak{V}_{A}(x)^{y}$, so that $d\left(x^{y}\right)=d(x)$. Hence, if $x$ and $y$ correspond to noncentral elements in the same subgroup of order $p^{2}$ of $G / A$, then $d(x)=d(y)$.

Let $K$ be the unique normal subgroup of $G$ with $K>A$ and $[K: A]=p$. Now $K=\langle A, w\rangle$ and we can assume that $w \in G^{\prime}$. Let $a_{0}=d(w)$ and let $a_{i}=d\left(x_{i}\right)$ for $x_{i} \in K_{i}-K$ where $K_{i}$ is the $i$-th normal subgroup of index $p$ in $G$ with $K_{i}>K$. There are clearly $p+1$ such subgroups.

Let $s(A)$ be the number of classes of conjugate characters of $A$ under the action of $G / A$ (see Definition 4.2 of [5]). Then by Lemma 4.3 (iv) of [5] we have

$$
|A|+(p-1)|A| / a_{0}+\left(p^{2}-p\right) \sum_{1}^{p+1}|A| / \alpha_{i}=[G: A] s(A) .
$$

Since $G$ has r.x.2, no character of $A$ can have more than $p^{2}$ conjugates and the principal character is fixed. Hence $s(A)>|A| / p^{2}$ and we obtain

$$
1 / a_{0}+p \sum_{1}^{p+1} 1 / a_{i}>1 \text {. }
$$


(3.6) Lemma. Let $G$ have r.x.2. Let $A$ be a normal elementary abelian q-subgroup such that $G / A$ is nonabelian of order $p^{3}$. Suppose that $G / A$ acts faithfully and irreducibly on $A$. Then $G \simeq G_{0}$ with $A$ corresponding to $V_{0}$ and so $p=2$ and $q=3$.

Proof. We use the notation given in the material directly preceding equations (*) and (**). Since $G / A$ is a nonabelian $p$-group we see that the number of generators of $A$ is divisible by $p$. Say $|A|=q^{r p}$. We have clearly $3(K)=1$, so that $a_{0}=|A|$.

Let $\lambda$ be a nonprincipal character of $A$. Since $G$ has r.x.2, $\lambda$ has $1, p$ or $p^{2}$ conjugates. If $\lambda$ had 1 or $p$ conjugates, then its inertia group would contain $K$. However, if $w$ fixes $\lambda$, we would have $1<(K, A) \subseteq \operatorname{ker} \lambda<A$ and then $(K, A)$ would be a nontrivial $G / A$ submodule of $A$, a contradiction. Hence $\lambda$ has $p^{2}$ conjugates. From this we conclude that the number of classes of conjugate characters of $A$ is equal to

$$
s(A)=1+(|A|-1) / p^{2} .
$$

Hence by $(*)$, since $a_{0}=|A|$, we have

$$
|A|+(p-1)+\left(p^{2}-p\right) \sum_{1}^{p+1}|A| / a_{i}=p^{3}\left\{1+(|A|-1) / p^{2}\right\}
$$

or

$$
p \sum_{1}^{p+1}|A| / a_{i}=p^{2}+p-1+|A| .
$$

Let $x$ and $y$ be elements of $K_{i}-K$ with $\langle A, x, y\rangle=K_{i}$. Then $w$ centralizes $\mathfrak{F}_{A}(x) \cap \mathfrak{F}_{A}(y)$. Since $w$ acts fixed point free on $A$ we have $\mathfrak{\complement}_{A}(x) \cap \mathfrak{\complement}_{A}(y)=1$, and hence $|A|$ divides $\alpha_{i}^{2}$. In particular $|A|^{1 / 2} \geqq|A| / a_{i}$. Then $(* * *)$ becomes

$$
p(p+1)|A|^{1 / 2} \geqq p^{2}+p-1+|A|
$$

or

$$
p(p+1) \geqq|A|^{1 / 2}+1=q^{r p / 2}+1 .
$$

Let us assume first that $p>2$. If $r=1$ then $G F(q)$ must contain the $p^{\text {th }}$ roots of unity. Hence $q>p$ and so $q \geqq p+2$. Then the above equation becomes

$$
\left(p^{2}+p-1\right)^{2} \geqq(p+2)^{p},
$$

a contradiction for $p \geqq 3$. Thus $r \geqq 2$ and

$$
p(p+1) \geqq q^{r p / 2}+1 \geqq q^{p}+1 \geqq 2^{p}+1 .
$$

The only possibility here is easily seen to be $p=3, q=2$ and $r=2$. But then $(* * *)$ becomes 


$$
3 \sum_{1}^{4}|A| / a_{i}=11+64=75
$$

and so

$$
\sum_{1}^{4}|A| / \alpha_{i}=25 .
$$

Now each $|A| / a_{i}$ is a power of two at most equal to $8=64^{1 / 2}$. Hence the only solution of the above is $|A| / a_{1}=1$ and $|A| / a_{i}=8$ for $i=$ $2,3,4$. If $G / A$ does not have period $p$ then only one subgroup of order $p^{2}$ is noncyclic. Thus, say $K_{2}$ is cyclic and since $a_{2}=8$ we have $\left[A: 3\left(K_{2}\right)\right]=8$, a contradiction. On the other hand, if $G / A$ has period $p$, then $K_{1} / A$ is an abelian group of type $(p, p)$ acting fixed point free on $A$, again a contradiction. Thus $p>2$ cannot occur.

Now let $p=2$. We have

$$
2\left(2+{ }^{\circ} 1\right)=6 \geqq q^{r}+1,
$$

so the only possibilities are $r=1$ and $q=3$ or 5 . If $q=5$ we have equality throughout and thus $a_{i}^{2}=|A|$ for all $i$. But a nonabelian group of order 8 always has a normal cyclic subgroup of order 4 . If this corresponds to $K_{1}$ then $\left[A: 3\left(K_{1}\right)\right]=5$ and $w$ fixes $3\left(K_{1}\right)$, a contradiction. This leaves only $q=3$. Here we have from $\left({ }^{* *}\right)$ the equation

$$
\sum_{1}^{3}|A| / \alpha_{i}=7,
$$

and so $|A| / a_{1}=1,|A| / a_{2}=|A| / a_{3}=3$. Since $K_{2} / A$ cannot be cyclic we see that $G / A$ is the dihedral group of order 8 . Thus we have $\Im_{2}(G) \simeq G / A$ the dihedral group acting on a $(3,3)$ group and this is the group $G_{0}$ already discussed. This completes the proof.

Proof of Proposition 3.3. We will have need here for Lemma 3.5 applied to $G$. If the conclusion of that result is $[G: 3(G)]=p^{6}$ we are trivially through. Hence we assume in the following that the conclusion is always $[3(K): 3(G)]=p$.

There are three distinct cases to study. Case 1 assumes that $G / A$ is abelian. In Case 2 we suppose $G / A$ is nonabelian and that $\mathfrak{g}(G)$ is central. Finally in Case 3 we consider the possibility that $G / A$ is nonabelian and that $\mathfrak{S}(G)$ is not central.

Case 1. We show first that $H=\mathfrak{S}(G)$ is central here. Write $A=H \times P$ where $P$ is the Sylow $p$-subgroup of $A . \quad P$ is characteristic in $A$ and hence normal in $G$. Consider $\bar{G}=G / P$. If $G$ has r.x.2, so does $\bar{G}$. Also $\mathfrak{S}_{p}(\bar{G}) \simeq G / A$ is abelian. Hence $\bar{G}$ has r.x.(2,0) (see Definition 3.1 of [5]). Then by Theorem A of [5] we see that $\bar{G}$ has an abelian subgroup $\bar{B}$ of index dividing $p^{2}$. Let $B$ be its complete inverse image in $G$. Clearly $B>A$, since $B \supseteqq H, B \supseteqq P$ and $[G: B]<[G: A]$. 
We show that $B$ centralizes $H$. Since $\bar{B}$ is abelian, we have $(B, H) \subseteq P$. But $H$ is normal so $(B, H) \subseteq H$. Since $H \cap P=1$ we have $(B, H)=1$. Hence $\sqrt{ }(H)>A$ and $\sqrt{ }(H)$ is normal in $G$. If $[G: \mathfrak{C}(H)] \leqq p$ then $H \subseteq 3(G)$ by Hypothesis 3.2. Finally, suppose $K=\mathbb{S}(H)$ has index $p^{2}$ in $G$. Since $A$ is self centralizing, $K$ is non-

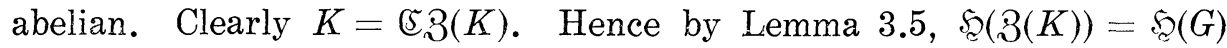
is central in $G$.

Since $\mathfrak{g}(G)$ is central, it clearly suffices to assume that $G$ is a $p$ group. Now $A=\subseteq(A)$ has index $p^{3}$ in $G$, so by Lemma 4.4 of [5] there exists $x \in G-A$ with $\left[A\right.$ : $\left.\mathfrak{C}_{A}(x)\right] \leqq p^{2}$ and $x^{p} \in A$. Hence $K=\langle A, x\rangle$ is normal, $[G: K]=p^{2}$ and $[K: 3(K)] \leqq p^{3}$. Moreover, $K$ is nonabelian. By Hypothesis 3.2 and Lemma 3.5 we have $[G: 3(G)] \leqq p^{6}$.

Case 2. Since $\mathfrak{S}(G)$ is central we can assume that $G$ is a $p$-group. We use the notation of equations $\left(^{*}\right)$ and $\left(^{* *}\right)$. Suppose $a_{0} \leqq p^{2}$. Then $[K: 3(K)] \leqq p^{3}$ and by Hypothesis 3.2 and Lemma 3.5 we have $[G: 3(G)] \leqq p^{6}$. Now suppose for some $i \neq 0, a_{i}=p$. Let $x, y \in K_{i}-K$ such that $K_{i}=\langle A, x, y\rangle$. Then $3\left(K_{i}\right) \supseteqq \mathfrak{\Im}_{A}(x) \cap \Im_{A}(y)$. But $3\left(K_{i}\right) \cong 3(G)$, so we have $[G: 3(G)] \leqq p^{5}$.

We assume now that $\alpha_{0} \geqq p^{3}$ and $\alpha_{i} \geqq p^{2}$ and obtain a contradiction. By Lemma 4.4 of [5] or by $\left({ }^{*}\right)$ above, some $a_{i} \leqq p^{2}$. Say $a_{1}=p^{2}$. Let $x$ and $y$ be elements of $K_{1}-K$ such that $K_{1}=\langle A, x, y\rangle$. Then $\mathfrak{F}_{A}(x) \cap \mathfrak{\Im}_{A}(y) \subseteq 3\left(K_{1}\right) \subseteq 3(G)$ by Hypothesis 3.2 , so $[A: 3(G)] \leqq p^{4}$.

Since $A>3(G)$ we see that $V=3_{2}(G) \cap A>3(G)$. Now by Lemma 1.2 (iii), $G^{\prime}$ centralizes $\bigotimes_{2}(G)$ so $\mathfrak{E}_{4}(w) \supseteqq \bigotimes_{2}(G) \cap A=V>3(G)$. Since $a_{0} \geqq p^{3}$ and $[A: 3(G)] \leqq p^{4}$, we see that $a_{0}=p^{3}, \quad[A: 3(G)]=p^{4}$ and $\widetilde{c}_{A}(w)=V$ with $[V: 3(G)]=p$. We show now that at least two of the $a_{i}$ are equal to $p^{2}$. First suppose $p>2$. If $a_{i} \geqq p^{3}$ for $i \neq 1$, we have by $(* *)$

$$
1 / p^{3}+p / p^{2}+p\left(p / p^{3}\right)>1
$$

or $1+2 p^{2}>p^{3}$, a contradiction. If $p=2$, then some $K_{i}$, say $K_{3}$, is such that $K_{3} / A$ is cyclic. Since $3\left(K_{3}\right)=3(G)$ we have $a_{3}=p^{4}=2^{4}$. Hence by $\left({ }^{* *}\right)$ again

$$
1 / 2^{3}+2 / 2^{2}+2 / a_{2}+2 / 2^{4}>1
$$

and $8=2^{3}>a_{2}$. Thus $a_{2}=2^{2}$. Say $a_{1}=a_{2}=p^{2}$.

Let $x \in K_{1}-K$ and $y \in K_{2}-K$ be chosen so that $G=\langle A, x, y\rangle$. Then $\left[A: \mathbb{E}_{A}(x)\right]=\left[A: \mathbb{E}_{A}(y)\right]=p^{2}$. Moreover $\mathfrak{E}_{A}(x) \nsupseteq V$ and $\mathfrak{E}_{A}(y) \nsupseteq V$. This follows since $\mathfrak{C}_{A}(x) \supseteqq V$ implies $V \cong 3(\langle A, x, w\rangle)=3\left(K_{1}\right)=3(G)$, a contradiction. Set $U=V \mathfrak{S}_{A}(x) \cap \mathfrak{E}_{A}(y)$. By the above we see that $U>3(G)$. Now $(y, U)=1 \leqq 3(G)$, since $U \subseteq \mathfrak{夭}_{A}(y)$ and $(x, U) \subseteq 3(G)$, since $U \subseteq V \complement_{A}(x)$ and $V \leqq \Re_{2}(G)$. But $G=\langle A, x, y\rangle$, so we see that 
$3(G)<U \subseteq A \cap \bigotimes_{2}(G)=V$ and hence $U=V$. This is a contradiction, since $V \nsubseteq \mathfrak{\complement}_{A}(y)$.

Case 3. If $\hat{g}(G)$ were centralized by $w$ it would be central by Lemma 3.5 and Hypothesis 3.2, which is not the case. Write $A=Q \times R$ where $Q$ is a Sylow $q$-subgroup of $A$ not centralized by $w$ and $R$ includes the remaining Sylow subgroups of $A$. Here, of course, $q \neq p$. Let $G_{1}=G / R$ with $Q_{1}=A / R \simeq Q$ and $w_{1}=w R / R$. Then $w_{1}$ does not centralize $Q_{1}$, since $(w, Q) \subseteq Q$. In $G_{1}$ let $\Phi\left(Q_{1}\right)$ be the Frattini subgroup of $Q_{1}$. Then $w_{1}$ does not fix $Q_{1} / \Phi\left(Q_{1}\right)$ by Theorem 12.2.2 of [2]. Set $G_{2}=G_{1} / \Phi\left(Q_{1}\right)$ and $Q_{2}=Q_{1} / \Phi\left(Q_{1}\right)$. Here we have $G_{2} / Q_{2} \simeq G / A$ acting faithfully on an elementary abelian $q$-group. Since $q$ is prime to $p$, the representation is completely reducible. One of the irreducible constituents will be faithful, otherwise $w_{2}=w_{1} \Phi\left(Q_{1}\right) / \Phi\left(Q_{1}\right)$ will be in all the kernels and hence centralize the group. Dividing $G_{2}$ by the product of the remaining representation spaces, we have a group $\bar{G}$ with r.x.2 with an elementary abelian $q$-subgroup $\bar{A}$ of index $p^{3}$ such that $\bar{G} / \bar{A}$ acts irreducibly and faithfully on $\bar{A}$. By Lemma 3.6 we must have $p=2, q=3,|\bar{A}|=9$ and $\bar{G} \simeq G_{0}$.

Thus in $G$ we have $p=2$ and $[A: 3(G)]$ is divisible by 9 . Moreover, $G / A$ is the dihedral group of order 8 . We apply equation $\left(^{* *}\right)$. Let $K_{1}$ correspond to the cyclic subgroup of order 4 of $G / A$. Then we have $a_{0} \geqq 9$ and $a_{1} \geqq 9$. Thus

$$
1 / 9+2 / 9+2 / a_{2}+2 / a_{3}>1
$$

or $1 / a_{2}+1 / a_{3}>1 / 3$. Hence one of $a_{2}$ or $a_{3}$ is less than 6 . Say $a_{2}<6$. Since $[A: 3(G)]$ divides $\alpha_{2}^{2}$ we have that 9 divides $\alpha_{2}^{2}$ and so $\alpha_{2}$ is divisible by 3 . Thus $a_{2}=3,[A: 3(G)]=9$ and $G / 3(G) \simeq G_{0}$. This completes the proof.

4. The main result. Our characterization of groups with r.x.2 is as follows.

(4.1) Theorem A. Group $G$ has $r . x .2$ if and only if $G$ satisfies one of the following.

(i ) $(p=2) G$ has subgroups $N$ and $K$ with

$$
G \underset{2}{\underbrace{}_{2}} N \underset{2}{\underbrace{}_{2}} K>3(K)>\underbrace{>}_{2} 3(N)>\underbrace{>}_{2} 3(G)>1
$$

such that $(G, 3(N)),(N, 3(K))$ and $K^{\prime}$ are the three distinct subgroups of order 2 of $U$, an abelian subgroup of type $(2,2)$ contained in $3(N)$.

(ii) $(p=2) G$ is a central extension of an abelian group $Z$ by $G_{0}$ such that $Z V_{0}$ is abelian. (Group $G_{0}$ is defined in Example 3.1).

(iii) $G$ has a normal abelian subgroup $A$ with index [G:A] 
dividing $p^{2}$.

(iv) $G$ has a normal subgroup $N$ of index $p$ with $[N: 3(N)]=p^{3}$.

(v) $[G: \mathfrak{Z}(G)]=p^{5}$.

(vi) $[G: 3(G)]=p^{6}$ and for all subgroups $N$ of $G$ of index $p$ with $G>N>3(G)$ we have $3(N)=3(G)$. Moreover, every homomorphic image of $G$ satisfies one of these six properties (i)-(iv).

It is interesting to note that the anomalous behavior of $p=2$ for groups with r.x.2 supports the conjecture that additional and more complicated groups with r.x.e can occur if $p \leqq e$ as compared to $p \geqq e+1$ (see Propositions 4.6 and 4.7 of [5]). This is analogous to the situation of regular $p$-groups in which for $p \geqq n$ all the groups of order $p^{n}$ are regular while for $p \leqq n-1$ there is at least one nonregular group.

We show by example that all of the cases of Theorem A occur independently of each other.

(i) Let $G=F_{3}$ be the group of Example 2.8.

(ii) Take $G=G_{0}$ here.

(iii) Set $G=F_{2} \times F_{2}$ where $F_{2}$ is the group of Example 4.5 of [5].

(iv) Take $G=F_{1} \times F_{2}$ where $F_{1}$ is the group of Example 3.7 of [5].

(v) Let $G$ be the following group of order $p^{15} . G$ is generated by elements $x_{i}(i=1,2,3,4,5)$ and $y_{i j}(i>j$ and $i, j=1,2,3,4,5)$, all of order $p$ such that for $i>j, y_{i j}=\left(x_{i}, x_{j}\right)$. Let $Z$ be the central subgroup generated by the $y_{i j}$. If $U$ is a subgroup of $G$ with $U>Z$ and $[U: Z]=p$, then it is not hard to see that $U=\mathfrak{E}(U)$. With this we see that $Z=3(G)$ and $G$ does not satisfy (iii) or (iv).

(vi) Set $G=F_{1} \times F_{1}$. Then $[G: 3(G)]=p^{6}$ and $G$ does not satisfy (i) or (ii). Since $F_{1}$ has r.x.1 we see that $G$ has r.x.2.

The proof of the sufficiency half of Theorem $A$ is simple and we do this first. Let $G$ satisfy one of (i)-(vi). By Ito's theorem the degrees of all characters of $G$ are powers of $p$. Cases (i) and (ii) follow from Proposition 2.7 and 3.3 respectively. Cases (iii) and (v) follow by Proposition 1.1 and 1.4 of [5]. In Case (iv) clearly $N$ has r.x.1. Let $\chi$ be a character of $G$ with $\chi \mid N=a \sum_{1}^{t} \varphi_{i}$. Then $\operatorname{deg} \chi=a t \operatorname{deg} \varphi_{1}$ so $a$ and $t$ are powers of $p$. But by Lemma 1.2 of [5], $a^{2} t \leqq[G: N]=p$, so $a=1$ and $t=1$ or $p$. Since $\operatorname{deg} \varphi_{1}=1$ or $p$, this yields the result.

Finally, given case (vi). By Proposition 1.4 of [5], $G$ has r.x.3. Let $\chi$ be a character of $G$ and let $\bar{G}$ be the homomorphic image of $G$ which is the faithful linear group associated with $\chi$. If $\bar{G}$ satisfies (i) $-(\mathrm{v})$, then $\bar{G}$ has r.x.2 and hence $\operatorname{deg} \chi$ divides $p^{2}$. The result will follow then if we show that $\bar{G}$ does not satisfy (vi). Now $G$ is nilpotent so therefore so is $\bar{G}$. Let $\zeta \in 3_{2}(\bar{G})-3(\bar{G})$ with $\zeta^{p} \in 3(\bar{G})$. Then the homomorphism $g \rightarrow(g, \zeta)$ maps $\bar{G}$ into $3(\bar{G})$, and the image has period $p$. Since $\bar{G}$ is a faithful irreducible linear group $3(\bar{G})$ is cyclic 
and so $|(\bar{G}, \zeta)|=p$. Hence $N=\mathfrak{E}(\zeta)$ has index $p$ in $\bar{G}$. Thus $\zeta \in 3(N)-3(\bar{G})$, so $\bar{G}$ cannot satisfy (vi).

(4.2) Lemma. Let $G$ have a normal subgroup of index $p$ with r.x.1. Then $G$ has one of the following.

(i) a characteristic abelian subgroup of index $p^{2}$;

(ii) a characteristic normal subgroup $N$ of index $p$ with $[N: 3(N)]$ dividing $p^{3}$;

(iii) a center of index dividing $p^{5}$.

Proof. Suppose first that $G$ has a normal abelian subgroup of index $p^{2}$. Let $K$ denote the group generated by all such subgroups. Clearly $K$ is a characteristic subgroup of $G$ and $[G: K]=1, p$ or $p^{2}$. If $[G: K]=p^{2}$ then $K$ is abelian and we have (i). If $[G: K]=p$ then $K=A_{1} A_{2}$ where $A_{1}$ and $A_{2}$ are normal abelian subgroups of $G$ of index $p^{2}$. Clearly $3(K) \geqq A_{1} \cap A_{2}$ and $\left[K: A_{1} \cap A_{2}\right]=p^{2}$, so we have (ii). Finally, suppose $K=G$. Write $G=A_{1} A_{2} A_{3}$ where $A_{1} A_{2}<G$. Then $\left[G: A_{1} \cap A_{2}\right]=p^{2}$ or $p^{3}$ and so $\left[G: A_{1} \cap A_{2} \cap A_{3}\right]$ divides $p^{5}$. Since $3(G) \geqq$ $A_{1} \cap A_{2} \cap A_{3}$ we have (iii).

Hence we can assume that $G$ has no normal abelian subgroup of index $p^{2}$. Let $N$ be the given normal subgroup of index $p$ with r.x.1. Since $N$ does not have a characteristic normal abelian subgroup of index $p$ we know by Lemma 1.1 (i) that $[N: 3(N)]$ divides $p^{3}$. If $N$ is characteristic we have (ii). If not, let $N_{1}$ be another normal subgroup of index $p$ with r.x.1. Now $N \cap N_{1}$ is normal and has index $p^{2}$ in $G$. Hence by assumption $N \cap N_{1}$ is nonabelian and we have $e\left(N \cap N_{1}\right)=$ $e(N)=e\left(N_{1}\right)=1$. By Lemma 3.4 (ii) (5 $3\left(N \cap N_{1}\right) \geqq\left\langle N, N_{1}\right\rangle=G$. But finally $N_{1} \cap 3(N) \subseteq 3\left(N \cap N_{1}\right)$ so we have (iii) again and the result follows.

Proof of Theorem A. We assume $G$ is nonabelian. If $G$ has a normal subgroup $N$ of index $p$ with r.x.1, then $G$ is type (iii), (iv) or (v) by Lemma 4.2. If $G$ has a normal subgroup $N$ of index $p$ with $e(N)=2$ and $(53(N)=N$ then $G$ is type (i) by Proposition 2.7. Thus it suffices to assume that $G$ has no subgroup of index $p$ with r.x.1 and that $G$ satisfies Hypothesis 3.2.

We use induction on $|G|$. Thus for all groups $\bar{G}$ of order less than that of $G$ having r.x.2 we assume one of the following.

(1) $\bar{G}$ has r.x.1;

(2) $\bar{G}$ has a normal subgroup $\bar{N}$ of index $p$ with r.x.1;

(3) $[\bar{G}: 3(\bar{G})]$ divides $p^{6}$;

(4) $\bar{G}$ is a central extension of abelian group $\bar{Z}$ by $G_{0}$ such that $\bar{Z} V_{0}$ is abelian.

We will show that either $[G: 3(G)]$ divides $p^{6}$ or $G$ has a normal 
abelian subgroup $A$ of index $p^{3}$. In the latter, since $G$ has no subgroup of index $p$ with r.x.1, $A$ must be self-centralizing. Then Proposition 3.3 will yield the theorem.

Now by Proposition 3.4 of [5], $G$ has a normal subgroup $N$ of index $p$. By assumption $e(N)=2$ so $N$ satisfies (2), (3) or (4) above. If $N$ satisfies (2), then the result follows by Lemmas 4.2 and 3.5, and Hypothesis 3.2. We assume then that $G$ has no subgroup of index $p^{2}$ with r.x.1.

Suppose $N$ satisfies (3). By Hypothesis 3.2 we see that $G$ is nilpotent. For convenience we can assume that $G$ is a $p$-group. We have $[G: 3(G)] \leqq p^{7}$. The only case we need consider is $[G: 3(G)]=p^{7}$ and we obtain a contradiction here. Let $J$ be a central subgroup of $G$ of order $p$ and set $\bar{G}=G / J$. Then $\bar{G}$ has r.x.2 and so satisfies (1), (2) or (3) above. Case (4) clearly cannot occur.

Suppose $\bar{G}$ is case (1). If $\bar{G}$ is abelian we have $[G: 3(G)] \leqq p^{4}$ by Lemma 1.3 of [5]. If $\bar{G}$ has a normal abelian subgroup $\bar{B}$ of index $p$, then let $B$ be its complete inverse image in $G$. As above $[B: 3(B)] \leqq p^{4}$. Since $3(B) \cong 3(G)$, this is also a contradiction. If neither of the two occur, then $[\bar{G}: 3(\bar{G})]=p^{3}$ by Lemma 1.1 (i). Let $\zeta$ belong to the complete image of $3(\bar{G})$ in $G$. Then $(G, \zeta) \subseteq J$ is, by Lemma 1.2 (iii), $[G:(\zeta))]=1$ or $p$. But by Hypothesis 3.2 we cannot have $[G: \subseteq(\zeta)]=p$. Thus $[G: 3(G)] \leqq p^{3}$, a contradiction.

Let $\bar{G}$ be case (2). Then either $\bar{G}$ has a normal abelian subgroup $\bar{B}$ of index $p^{2}$ or $\bar{G}$ has a normal subgroup $\bar{L}$ of index $p$ with $[\bar{L}: 3(\bar{L})]=p^{3}$. In the first case let $B$ be the complete inverse image of $\bar{B}$. Since $e(B)=2$, we have by Lemma 1.3 of [5] that $[B: 3(B)]=p^{4}$. Hence $[3(B): 3(G)]=p$ and we can choose $\zeta \in 3(B)-3(G)$. In the second case let $L$ be the complete inverse image of $\bar{L}$ and let $\zeta$ be the inverse image of $3(\bar{L})$ such that $\zeta \in B_{2}(G)-3(G)$ and $\zeta^{p} \in 3(G)$. (See Lemma 1.2 (ii).) Since $(\zeta, L) \leqq J$, we see that $\left[L: \widetilde{\mho}_{L}(\zeta)\right] \leqq p$. Thus in both cases we have an element $\zeta \in 3_{2}(G)-3(G)$ with $\zeta^{p} \in 3(G)$ and $\left[G:(\zeta(\zeta)] \leqq p^{2}\right.$. Clearly we must have $\left[G:(\zeta(\zeta)]=p^{2}\right.$. Now $G /(\zeta(\zeta) \simeq(G, \zeta)$ is elementary abelian of order $p^{2}$. Choose two distinct subgroups $G_{1}$ and $G_{2}$ with $G>G_{i}>\sqrt{ }(\zeta)$. Now $e\left(G_{i}\right)=e(\sqrt{ }(\zeta))=2$ and $\sqrt{5} 3(\mathbb{E}(\zeta))=\sqrt{ }(\zeta)$. Hence by Proposition 2.7, $\left(G_{1}, \zeta\right)=\left(G_{2}, \zeta\right)=\Omega(\mathbb{E}(\zeta))$. Thus $(G, \zeta)=$

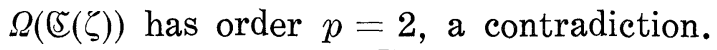

Finally, suppose $\bar{G}$ satisfies (3). Clearly the inverse image in $G$ of $3(\bar{G})$ is central and this contradicts $[G: 3(G)]=p^{7}$.

We need only assume now that $N$ satisfies (4). Since $V_{0}$ is a characteristic subgroup of $G_{0}$ we see that $A=V_{0} 3(N)$ is characteristic in $N$ and hence normal in $G . A$ is abelian and $G / A$ has order $p^{4}$ for $p=2$. By Lemma $1.3, G / A$ has a normal abelian subgroup of index $p$. Let $K$ be its complete inverse image in $G$. Then $K$ is metabelian and hence cannot be case (4), since clearly $G_{0}$ is not metabelian. Thus 
$K$ is one of cases (2) or (3), and the result follows. This completes the proof of Theorem A.

5. Corollaries. With the complete characterization of groups with r.x.2 we can easily read off several properties they have in common.

(5.1) Corollary. Let G have r.x.2. Then G has a normal abelian subgroup whose index divides $p^{4}$. In particular $f(2)=4$ where $f$ is the function studied in Theorem A of [5].

Proof. The result is clear for groups of type (ii) and (iii). If $G$ is type (iv) then since $G / 3(N)$ is a p-group and $N / 3(N)$ is normal, we can choose normal subgroup $A$ with $G>N>A>3(N)$ and $[A: 3(N)]=p$. Clearly $A$ is abelian. If $G$ is type (v) we merely take $A$ to be a normal subgroup with $A>3(G)$ and $[A: 3(G)]=p$.

Now let $G$ satisfy (i) or (vi). Suppose first that $G / 3(G)=J$ is not elementary abelian. By Lemma 1.4 we can find $\bar{g} \in 3(J)$ and $\bar{h} \in J^{\prime}$ so that $\langle\bar{g}, \bar{h}\rangle$ is a normal subgroup of $J$ of order $p^{2}$. Let $g$ and $h$ be inverse image of these elements of $G$ with $h \in G^{\prime}$. Set $A=\langle 3(G), g, h\rangle$. Then $A$ is a normal subgroup of $G$ of index $p^{4}$. But $g \in 3_{2}(G)$, so $g$ and $h$ commute by Lemma 1.2 (iii). Hence $A$ is abelian and the result follows here.

We need only consider the case where $J$ is elementary abelian. Suppose for some $x \in 3(G)$ we have $\subseteq(x)\rangle\langle x, 3(G)\rangle$. Then choose $y \in \mathfrak{S}(x)-\langle x, 3(G)\rangle$. Clearly $A=\langle x, y, 3(G)\rangle$ is an abelian subgroup of $G$ of index $p^{4}$ which is normal since $G / 3(G)$ is abelian. We suppose now that for all $x \notin 3(G)$ we have $\subseteq(x)=\langle x, 3(G)\rangle$ and obtain a contradiction. This will yield the result. With this information we have clearly that the number of classes $c$ of $G$ is given by

$$
\begin{aligned}
c & =|3(G)|+(|G|-|3(G)|) / p^{5} \\
& =|G|\left(1 / p^{5}+1 / p^{6}-1 / p^{11}\right) .
\end{aligned}
$$

Since $G$ has r.x.2 we have, using the fact that $G$ has $c$ characters, the inequality

$$
|G| \leqq c p^{4}=|G|\left(1 / p+1 / p^{2}-1 / p^{7}\right) .
$$

This is the required contradiction.

That $p^{4}$ is the best possible bound can be seen by considering the group $G=F_{1} \times F_{1}$ where $F_{1}$ is the group of Example 3.7 of [5].

(5.2) Corollary. Let $G$ have r.x.2. Then G has a normal subgroup $H$ of index dividing $p^{2}$ with $[H: 3(H)]$ dividing $p^{4}$ or 18 if $p=2$. 
This follows quite easily. It is possible that the $p^{4}$ term here can be reduced to $p^{3}$. This would require a further study of groups of type (vi).

The author would like to take this opportunity to thank his advisor Professor Richard Brauer for the help and encouragement he so freely gave.

\section{REFERENCES}

1. W. Burnside, Theory of groups of finite order, 2nd ed., Dover, New York, 1955.

2. M. Hall, Jr., Theory of groups, Macmillan, New York, 1959.

3. I. M. Isaacs and D. S. Passman, Groups with representations of bounded degree, Canad. J. Math. 16 (1964), 299-309.

4. __ Groups whose irreducible representations have degrees dividing $p^{e}$, Illinois J. Math. 8 (1964), 446-457.

5. - A characterization of groups in terms of the degrees of their characters, Pacific J. Math. 15 (1965), 877-903.

University of CAlifornia, Los ANgeles 


\title{
PACIFIC JOURNAL OF MATHEMATICS
}

\author{
EDITORS
}

\section{H. SAMELSON}

Stanford University

Stanford, California

R. M. Blumenthal

University of Washington

Seattle, Washington 98105

\section{*J. DugundJI}

University of Southern California Los Angeles, California 90007

\section{RichaRd ARENS}

University of California

Los Angeles, California 90024

\section{ASSOCIATE EDITORS}
E. F. BECKENBACH
B. H. NEUManN
F. WOLF
K. YOSIDA

\section{SUPPORTING INSTITUTIONS}

\author{
UNIVERSITY OF BRITISH COLUMBIA \\ CALIFORNIA INSTITUTE OF TECHNOLOGY \\ UNIVERSITY OF CALIFORNIA \\ MONTANA STATE UNIVERSITY \\ UNIVERSITY OF NEVADA \\ NEW MEXICO STATE UNIVERSITY \\ OREGON STATE UNIVERSITY \\ UNIVERSITY OF OREGON \\ OSAKA UNIVERSITY \\ UNIVERSITY OF SOUTHERN CALIFORNIA
}

\author{
STANFORD UNIVERSITY \\ UNIVERSITY OF TOKYO \\ UNIVERSITY OF UTAH \\ WASHINGTON STATE UNIVERSITY \\ UNIVERSITY OF WASHINGTON \\ AMERICAN MATHEMATICAL SOCIETY \\ CHEVRON RESEARCH CORPORATION \\ TRW SYSTEMS \\ NAVAL ORDNANCE TEST STATION
}

\footnotetext{
Mathematical papers intended for publication in the Pacific Journal of Mathematics should be typewritten (double spaced). The first paragraph or two must be capable of being used separately as a synopsis of the entire paper. It should not contain references to the bibliography. Manuscripts may be sent to any one of the four editors. All other communications to the editors should be addressed to the managing editor, Richard Arens at the University of California, Los Angeles, California 90024 .

50 reprints per author of each article are furnished free of charge; additional copies may be obtained at cost in multiples of 50 .
}

The Pacific Journal of Mathematics is published monthly. Effective with Volume 16 the price per volume (3 numbers) is $\$ 8.00$; single issues, $\$ 3.00$. Special price for current issues to individual faculty members of supporting institutions and to individual members of the American Mathematical Society: $\$ 4.00$ per volume; single issues $\$ 1.50$. Back numbers are available.

Subscriptions, orders for back numbers, and changes of address should be sent to Pacific Journal of Mathematics, 103 Highland Boulevard, Berkeley 8, California.

Printed at Kokusai Bunken Insatsusha (International Academic Printing Co., Ltd.), No. 6, 2-chome, Fujimi-cho, Chiyoda-ku, Tokyo, Japan.

PUBLISHED BY PACIFIC JOURNAL OF MATHEMATICS, A NON-PROFIT CORPORATION

The Supporting Institutions listed above contribute to the cost of publication of this Journal, but they are not owners or publishers and have no responsibility for its content or policies.

* Paul A. White, Acting Editor until J. Dugundji returns. 


\section{Pacific Journal of Mathematics}

\section{Vol. 17, No. $3 \quad$ March, 1966}

Tsuyoshi Andô, Contractive projections in $L_{p}$ spaces ............... 391

Robert F. Brown, On a homotopy converse to the Lefschetz fixed point theorem ............................................... 407

Richard Albert Cleveland and Sandra Cleveland, On the multiplicative extension property................................ 413

Harold H. Johnson, An algebraic approach to exterior differential systems..................................... 423

Alan Cecil Lazer, The behavior of solutions of the differential equation

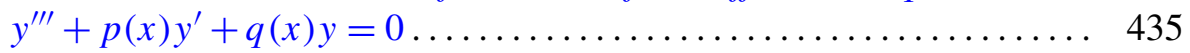

Judy Parr, Cohomology of cyclic groups of prime square order ......... 467

Donald Steven Passman, Groups whose irreducible representations have

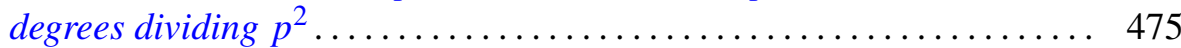

Ralph Tyrrell Rockafellar, Characterization of the subdifferentials of convex functions ........................................... 497

Donald Erik Sarason, Invariant subspaces and unstarred operator algebras...................................... 511

Donald Erik Sarason, Weak-star generators of $H^{\infty} \ldots \ldots \ldots \ldots \ldots \ldots . \ldots . \ldots$

Boris M. Schein, Homomorphisms and subdirect decompositions of semi-groups

Daniel Francis Shea, Jr., Functions analytic in a finite disk and having asymptotically prescribed characteristic

Zvi Ziegler, Generalized convexity cones 